\title{
Application of phase-metric compensation method for geoelectric control of near-surface geodynamic processes
}

\author{
Kuzichkin O. R. ${ }^{1}$, Vasilyev G. S. ${ }^{2}$, Grecheneva A. V. ${ }^{3}$, Mikhaleva E. V. ${ }^{4}$, Baknin M. D. ${ }^{5}$, Surzhik D. I. \\ ${ }_{1,2,3,4,5}$ Belgorod State University, Russia \\ ${ }^{6}$ Vladimir State University, Russia
}

\section{Article Info \\ Article history: \\ Received Aug 21, 2019 \\ Revised Dec 26, 2019 \\ Accepted Feb 28, 2020}

\section{Keywords:}

Compensation signal

Geodynamic monitoring

Karst processes

Multi-pole electrical installation

Phase-metric method

\begin{abstract}
This work is devoted to the application of the compensation method of geoelectric control using the field phase characteristics for the detection and localization of geodynamic processes. High noise immunity of the phase-metric registration method of geoelectric signals in comparison with amplitude parameters of the anomalous components of electromagnetic field, usually used to analyze the results of observations, is noted. A formalized approach to the use of phase characteristics of the field for the interpretation of monitoring data and associated problem of geodynamic processes localization is developed. In the framework of this approach, the section parameters are proposed to determine by the minimum sum of weighted mean square interpretation error and regularizing functional containing a priori information about the geoelectric section. To check localization possibility of the spherical karst-suffusion cavity, simulation of the amplitude and phase anomalous components of the field potential, as well as the standard error of heterogeneity localization, when moving the sphere center along the installation profile, is carried out. Simulation has shown a good potential differentiation from the inhomogeneity position, the highest accuracy of localization is achieved with combined use of amplitude and phase field components in the problem of inhomogeneity localization.
\end{abstract}

This is an open access article under the CC BY-SA license.

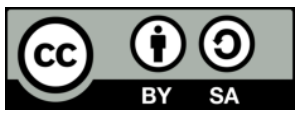

\section{Corresponding Author:}

Kuzichkin O. R.,

Belgorod State University,

85 Pobedy St, Belgorod, Russia.

Email: bakhteeyar@gmail.com

\section{INTRODUCTION}

Use of multipolar electrical installations in the problems of low-depth geophysical observation and control allows effective investigating and monitoring the local manifestations of geodynamic processes. At the same time, resolution of this method in geodynamic control significantly exceeds the capabilities of classical electric prospecting methods with a small number of emitting and receiving electrodes-vertical electric sounding and vertical electric profiling [1]. Disadvantage of using a large number of poles is the complexity of the measurement process, since each electrode in the course of observations needs to be moved separately. Technique of electrotomography (resistivity imaging, electrical resistivity tomography) can repeatedly use the same positions of the supply and measurement electrodes fixed on the observation profile. This leads to a decrease in the total number of operating positions of electrodes with significant increase in the density of measurements compared to the conventional method of vertical electrical sounding [2,3]. Currently, there is a wide class of electrotomography devices, manufacturers of which are, in particular, Phoenix Geophysics, Iris Instruments, R\&D enterprise "ERA", research Institute "GeoTech", "North-West". However, for application in the problems of geodynamic control organization their essential 
adaptation directed to the decrease of influence of natural and technogenic destabilizing factors on measurement results is necessary (according to requirements of normative documents of geotechnical monitoring organization [4]).

Developed algorithms for compensation of temperature noise, multiplicative noise of natural character and industrial noise in geoelectric control systems can reduce the noise value by 40-60 dB and increase the signal-to-noise ratio, which increases the probability of correct detection of negative geodynamics or reduces the probability of false alarm. To compensate temperature noise, the efficiency of regression processing of daily temperature deviations was proved [5-7]. To reduce the influence of meteorological factors of different nature, it is proposed to use automatic calibration of the ins tallation [8] and compensation of the geodynamic trend [9].

Efficiency of vector measurement systems with registration of phase characteristics of electromagnetic field, which are the basis of the phase-metric method of geoelectric control with high noise immunity [10], should be highlighted. This is due to the fact that the phase parameters of electromagnetic field are less affected by various noise compared to the amplitude parameters of the anomalous component of electromagnetic field, usually used to analyze the results of observations [11]. The use of specialized software in conjunction with electrotomography equipment allows interpretation of observation data in the scope of two-dimensional and three-dimensional models. At the same time, algorithms and programs aimed at solving the problems of interpretation of geoelectric control results based on compensation methods have not been developed at the moment. In particular, there is no formalized approach to the use of the field phase characteristics for interpretation of monitoring data and associated problem of geodynamic processes localization. The aim of the work is to substantiate application of the geoelectric control compensation method using the field phase characteristics for detection and localization of geodynamic processes on the example of the development of the karst-suffusion cavity.

\section{METHODOLOGY}

Phase-metric recording principle of geoelectrical data of geodynamic control. Work [10] explains application of the phase-metric method of geoelectric control, namely using multiple sources as the probing signal located in close proximity to the investigated object, and required number of vector measuring sensors of the electric field. In the simplest case, two point sources A, B and one measuring s ensor O located along the $\mathrm{AB}$ line and at equal distances from the sources can be used as shown in Figure 1(a).

When using a quadrature source of the probing field, sources A and B form probing signals shifted in phase by $\pi / 2$ relative to each other. It should be noted that with a different location of sources relative to the sensor, as well as with multi-pole sensing, phase shifts between the test signals may be different. Each of the point sources forms at the point $\mathrm{O}$ an electric field signal of the following form:

$$
\begin{aligned}
& \vec{E}_{A x}=\vec{E}_{A x}^{0}+\Delta \bar{E}_{A x}, \vec{E}_{A y}=\Delta \bar{E}_{A y} \\
& \vec{E}_{B x}=\vec{E}_{B x}^{0}+\Delta \bar{E}_{B x}, \vec{E}_{B y}=\Delta \bar{E}_{B y}
\end{aligned}
$$

where $\vec{E}^{0}$ is a normal signal in the absence of heterogeneity; $\Delta \bar{E}$ is an anomalous electric field component caused by the presence and geodynamics of heterogeneity.

As a result of the superposition principle of sources and fields the resulting normalized signal at the point $\mathrm{O}$, in accordance with Figure 1(b), has the form:

$$
\begin{aligned}
& H_{x}=\left(E_{A x}^{0} / I_{0}+\Delta E_{A x} / I_{0}\right) \sin (\omega t)+\left(E_{B x}^{0} / I_{0}+\Delta E_{B x} / I_{0}\right) \sin (\omega t+\varphi), \\
& H_{y}=\Delta E_{A y} \sin (\omega t) / I_{0}+\Delta E_{B x} \sin (\omega t+\varphi) / I_{0}
\end{aligned}
$$

A distinctive feature of this method is that the information recorded parameter is not amplitude, but phase $\varphi_{x, y}$ of the resulting signal, determined in accordance with the following relations at $\varphi=\pi / 2$ :

$$
\phi_{x}=\operatorname{arctg}\left(\frac{E_{A x}^{0}+\Delta E_{A x}}{E_{B x}^{0}+\Delta E_{B x}}\right), \phi_{y}=\operatorname{arctg}\left(\frac{\Delta E_{A y}}{\Delta E_{B y}}\right)
$$




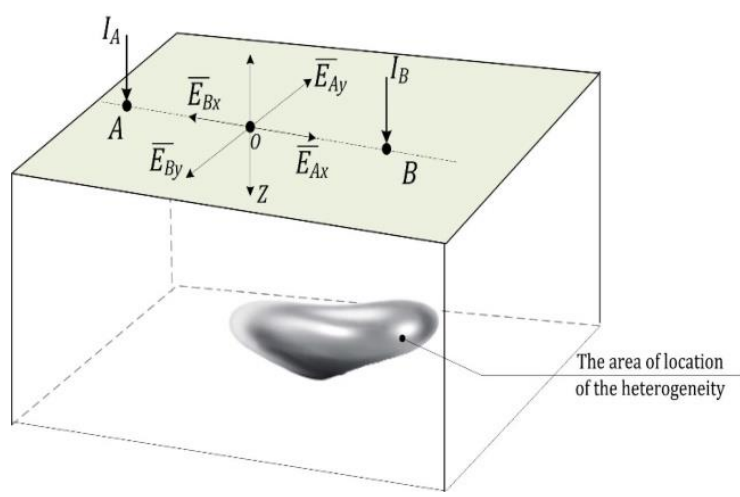

(a)

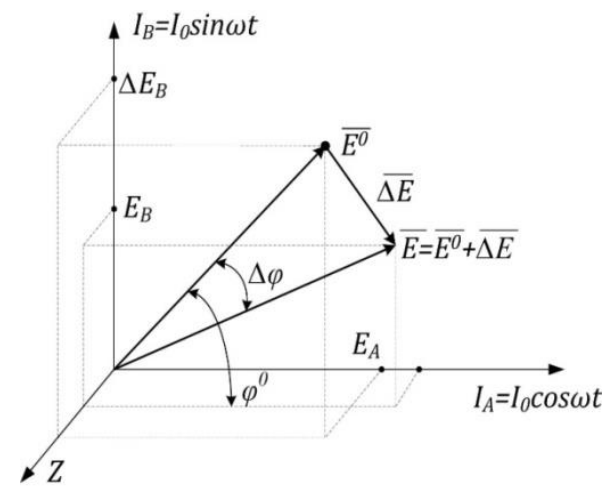

(b)

Figure 1. Phase-metric principle of geodynamic control

Geodynamic variations of the studied object are determined by displacement of fictitious sources, which leads to an imbalance of the measuring system and registration of the corresponding signal vector in it. The phase-metric registration method of geoelectric data is used to monitor geodynamics of near-surface inhomogeneities in cases of the need to provide increased sensitivity to special changes in the object of study. High efficiency is achieved by increasing sensitivity of the measuring system, initial installation and operational positioning of the electrical installation by controlling the sources of probing signals [11, 12].

Dynamic control mode of positioning of the phase-metric geoelectric control systems. Algorithms for positioning control of the phase-metric measuring geoelectric systems, as well as information processing, are based on the assumption that geodynamics of an object can be determined with a sufficient degree of accuracy by the results of field registration at $\mathrm{N}$ points with coordinates $\left(x_{i}, y_{i}\right)$ in the presence of $\mathrm{M}$ sensing points [11]. In accordance with superposition principle, a vector electric field signal of the following type is generated at each i-th measurement point by the source $\mathrm{j}$ :

$$
\begin{aligned}
& \bar{e}_{x i j}=\left[\psi_{i j}^{x}+G_{j}(j \omega) \sum_{k=1} \beta_{i j k}^{x}\right] I_{j} \exp \left(j \varphi_{j}\right), \\
& \bar{e}_{y i j}=\left[\psi_{i j}^{y}+G_{j}(j \omega) \sum_{k=1} \beta_{i j k}^{y}\right] I_{j} \exp \left(j \varphi_{j}\right)
\end{aligned}
$$

where $\psi_{i j}^{x}, \psi_{i j}^{y}$ are relative position spatial functions of the probing signal source and measuring sensors; $\beta_{i j k}^{x}, \beta_{i j k}^{y}$ are relative position spatial functions of the imaginary source $\mathrm{k}$, determined by anomalous geodynamics of the object under study; $I_{j}, \varphi_{j}$ are amplitude and phase of the probing signal; $\mathrm{Gj}(j \omega)$ is a contrast ratio of the imaginary sources.

The spatial functions in (4) can be determined at the initial system initialization by the method of sequential activating of probing signals at the selected poles. To ensure high sensitivity of system to small geodynamic changes of the object, it is necessary to carry out the initial installation and balance it on the recorded electric field vectors. This will eliminate from the recorded signals the constant component determined by initial inaccurate installation of the probing signal sources and natural misalignment of electrolocation signals from the object under study. The balancing condition can be defined from the expression $\bar{e}_{x}=\bar{e}_{y}=0$.

The principle of the system dynamic control is based on the constant adjustment of system due to phase control of probing signals. Under condition of initial positioning and that the probing signals of multipolar phase-metric systems can be represented with high accuracy either by single harmonic oscillations with certain amplitudes, frequencies and phases, or by a set of such oscillations:

$$
\begin{aligned}
& \Delta \bar{e}_{x i}(j \omega)=G_{i}(j \omega) \sum_{j=1} \sum_{k=1} \Delta \beta_{i j k}^{x} \Delta U_{j}(j \omega), \\
& \Delta \bar{e}_{y i}(j \omega)=G_{i}(j \omega) \sum_{j=1} \sum_{k=1} \Delta \beta_{i j k}^{y} \Delta U_{j}(j \omega)
\end{aligned}
$$

When using a reference quadrature generator in the formation of probing signals of a multipolar installation: 


$$
\Delta I_{j}(j \omega)=\Delta I_{a j} \sin \omega t+\Delta I_{b j} \cos \omega t
$$

where $\Delta I_{a j}=\Delta I_{j} \cos \phi_{j}, \Delta I_{b j}=\Delta I_{j} \sin \phi_{j}$ are amplitudes of the source j quadrature components. Based on (3), we obtain phase geodynamic displacements at the registration points of geoelectric field:

$$
\begin{array}{r}
\Delta \phi_{x i} \approx \frac{\operatorname{Re}\left(G_{i}(j \omega)\right) \sum_{j=1} \sum_{k=1} \Delta \beta_{i j k}^{x} \Delta I_{b j}+\operatorname{Im}\left(G_{i}(j \omega)\right) \sum_{j=1} \sum_{k=1} \Delta \beta_{i j k}^{x} \Delta I_{a j}}{\operatorname{Re}\left(G_{i}(j \omega)\right) \sum_{j=1} \sum_{k=1} \Delta \beta_{i j k}^{x} \Delta I_{a j}-\operatorname{Im}\left(G_{i}(j \omega)\right) \sum_{j=1} \sum_{k=1} \Delta \beta_{i j k}^{x} \Delta J_{b j}}, \\
\Delta \phi_{y i}=\frac{\operatorname{Re}\left(G_{i}(j \omega)\right) \sum_{j=1} \sum_{k=1} \Delta \beta_{i j k}^{y} \Delta I_{b j}+\operatorname{Im}\left(G_{i}(j \omega)\right) \sum_{j=1} \sum_{k=1} \Delta \beta_{i j k}^{y} \Delta I_{a j}}{\operatorname{Re}\left(G_{i}(j \omega)\right) \sum_{j=1} \sum_{k=1} \Delta \beta_{i j k}^{y} \Delta I_{a j}-\operatorname{Im}\left(G_{i}(j \omega)\right) \sum_{j=1} \sum_{k=1} \Delta \beta_{i j k}^{y} \Delta I_{b j}}
\end{array}
$$

Detection and localization of near-surface geodynamic process on the example of karst-suffusion cavity. Problem of interpretation of the parameters of geoelectric section based on the observation of several parameters of electric field using a multipolar installation can be defined in general as:

$$
\rho(\mathbf{r})=\arg \min \sum_{\Lambda} w_{\Lambda} \sigma_{\Lambda}^{2}+\alpha\|\boldsymbol{\Omega}\|^{2}
$$

where $\rho$ is resistivity, $r=\left(\begin{array}{lll}x & y & z\end{array}\right)^{T}$ is a vector of coordinates of a small fragment of geological medium, $\Lambda$ is an observed parameter of electric field (potential or field strength, as well as their amplitude or phase of its longitudinal or transverse component), $w_{\Lambda}$ is a weight coefficient of the parameter, $\sigma_{\Lambda}$ is a mean square error (MSE) on the interpretation parameter, $\alpha$ is a regularization parameter; $\|\boldsymbol{\Omega}\|^{2}$ is quadratic norm of the regularizing functional containing a priori information about the geoelectric section. MSE of interpretation by parameter $\Lambda$ is determined by the expression:

$$
\sigma_{\Lambda}^{2}=\frac{1}{M-1} \sum_{m=1}^{M} w_{i j}\left(\Lambda_{i}-\tilde{\Lambda}_{i}\right)^{2}
$$

where $\mathrm{M}$ is a number of registration points of geoelectric field, is a weight of the registration point of geoelectric field with respect to the field source $\mathrm{j}$, and is an exact and approximate value of the field parameter at the receiving point, respectively.

Including weight coefficients is necessary, since in practice it is often difficult or impossible to ensure a uniform distribution of electromagnetic field registration points during geodynamic studies. This is due to the fact that most geodynamic objects are located in areas of complex development, typical for most industrial enterprises or in conditions of limited access. When solving the problems of geodyn amic control, collection system used is initially stationary and uneven. Due to the fact that distances from the object and to the source of the probing signal are initially different, registration errors of electromagnetic field at different observation points have different variances. Therefore, a distinctive feature of the application of processing methods in geodynamic measuring systems, which allows to significantly reduce the error of geodynamic estimates, is the use of weight coefficients [13-20]. Weight coefficients are determined based on the dependence of the variance of approximation errors of the field parameter on the argument, and accordingly determined by localization of the field registration point $r i$ in relation to the probing field source $r j$ and the object of study:

$$
w_{i j}=1 / L_{j}\left(\mathbf{r}_{i}\right), \sum_{n=1}^{N} \sum_{m=1}^{M} w_{m, n}=1
$$

Interpretation problems of geological section parameters in general, and localization of heterogeneity in particular, are ill-posed, therefore, application of regularization by A. N. Tikhonov is necessary [14, 15]. In addition, the problem of lack of a priori information about the section for the construction of regularizing functional $\Omega$, characteristic for both electrical prospecting and monitoring observations, should be noted. This forces the use of additional simplifying assumptions about the nature of the localized heterogeneity. In particular, distribution of the point electrode field in the presence of a karst suffusion cavity is conveniently described by a model of spherical inhomogeneity [21-26]: 


$$
\dot{U}(r, \theta)=\frac{\dot{I} \rho_{1}}{4 \pi d} \sum_{n=0}^{\infty}\left(\frac{R}{d}\right)^{n} P_{n}(\cos \theta)+\sum_{n=0}^{\infty} A_{n} R^{n} P_{n}(\cos \theta) A_{n}=\frac{\dot{I} \rho_{1}}{4 \pi} \frac{\left(\rho_{2}-\rho_{1}\right) n}{\left(\rho_{1} n+\rho_{2}(n+1)\right) d^{n+1}}
$$

where is a Legendre polynomial; is a cavity radius; is distance from the sensing point to the center of inhomogeneity; is an angle between the direction from the center of inhomogeneity at the point of phase control with respect to the direction at the sensing point; coefficient is determined by boundary conditions and spatial parameters of subsurface heterogeneity as shown in Figure 2. Also geodynamic changes of cavity parameters are designated in Figure 2 as $\Delta \mathrm{d}, \Delta \theta, \Delta \mathrm{h}$.

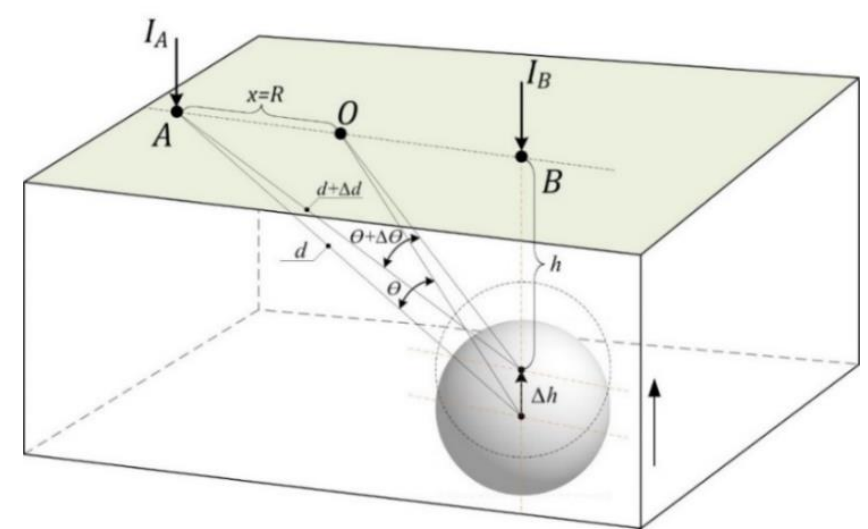

Figure 2. Calculation model of karst-suffusion process

\section{SIMULATION RESULTS}

To verify localization possibility of karst-suffusion cavity, simulation of the interpretation procedure of the section parameters according to the expression (7) has been done. The model parameters were set: installation base $\mathrm{L}=\mathrm{AB}=100 \mathrm{~m}$, probe frequency $\mathrm{f}=72 \mathrm{~Hz}$, electrical parameters of the host medium (moderately moist sand) $\rho 1=100 \mathrm{Ohm} \cdot \mathrm{m}$ and $\varepsilon 1=20$, electrical parameters of the karst cavity $\rho 2=1000$ $\rho 1=100 \mathrm{kOhm} \cdot \mathrm{m}$ and $\varepsilon 2=1$, cavity depth $\mathrm{h}=10 \mathrm{~m}$, cavity radius $\mathrm{R}=3 \mathrm{~m}$. Distance from the source $\mathrm{A}$ to the sphere center along the earth's surface was taken to be $\mathrm{xl}=20 \mathrm{~m}$. Distance $\mathrm{x} 0=\mathrm{AO}$ varied $(30$ and $50 \mathrm{~m})$, which is important for complex construction conditions, when the use of a symmetrical installation $\mathrm{AO}=\mathrm{OB}=\mathrm{L} / 2$ is impossible. Amplitudes of the sensing currents were set by expressions $\mathrm{IA}=1 \mathrm{~A}$ and to fulfill the balance condition of installation in its initial state (in the absence of a karst cavity).

Figure 3 shows changes in amplitude (Figure 3(a)) and phase (Figure 3(b)) of the anomalous field potential component when moving the sphere center along the installation profile from $\mathrm{x}=0$ to $\mathrm{x}=\mathrm{L}$ for different values of phase shifts between the probing signals $\varphi A B=00 ; 450: 1350 ; 900 ; 1800$. Simulation has shown a good potential differentiation from the location of heterogeneity. As can be seen from Figure 3(a), there is an ambiguity of the potential amplitude on $\mathrm{x}$, which forces the use, along with the amplitude component, also the potential phase component. At $\varphi \mathrm{AB}=00$ and 1800 phase differences are manifested only in the polarity of the received signal (Figure 3(b)).

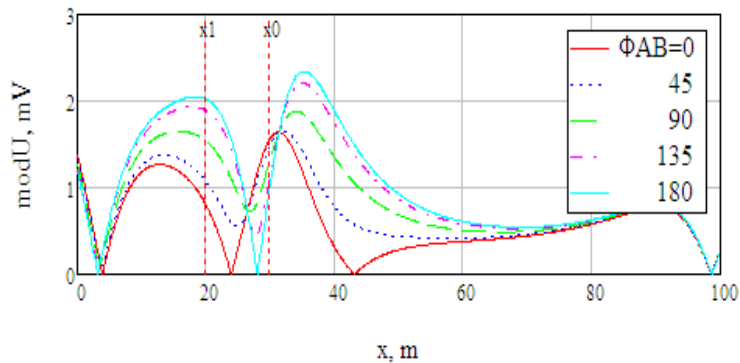

(a)

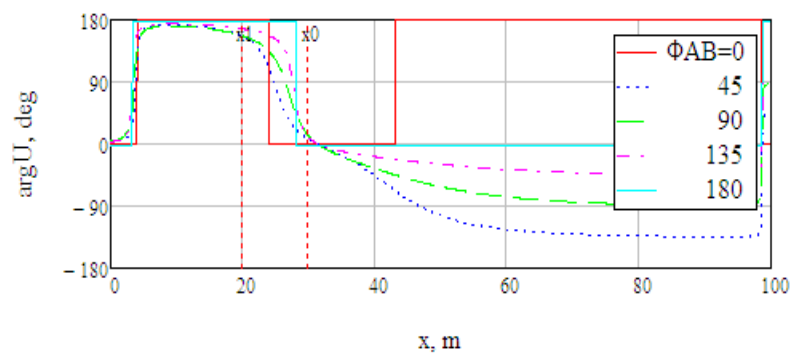

(b)

Figure 3. Changes in amplitude, (a) Phase, (b) The anomalous field potential component when moving the sphere center along the installation profile 
Figure 4 shows MSE change of the inhomogeneity localization at its displacement along the profile with respect to the true position $\mathrm{xl}=20 \mathrm{~m}$. Localization error is simulated for different weight coefficients of the amplitude component (module) of the field $\mathrm{w}_{-} \bmod =1 ; 0.5 ; 0$ and the phase component w_phase $=1-$ w_mod. Best results for both symmetric (Figure 4(a) and 4(b)) and for asymmetric installation (Figure 4(c)), characterized by absence of local minima and the smallest global minimum region, obtained with simultaneous equal use of the amplitude and phase components $w_{-}$mod=wphase $=0.5$. It should be noted that for another geoelectric sections or another types of probing installations, the optimal weight coefficients may be different.

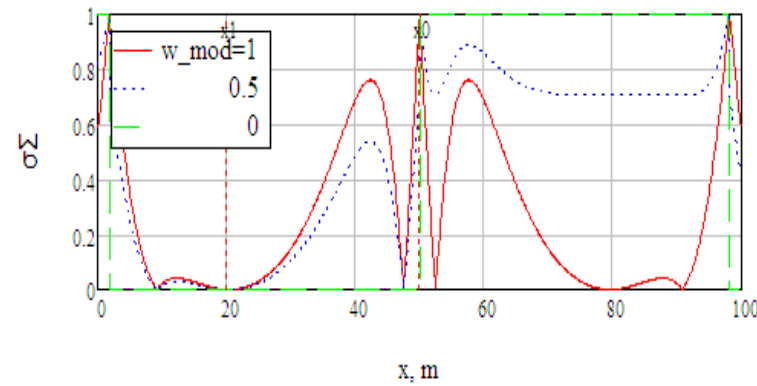

(a)

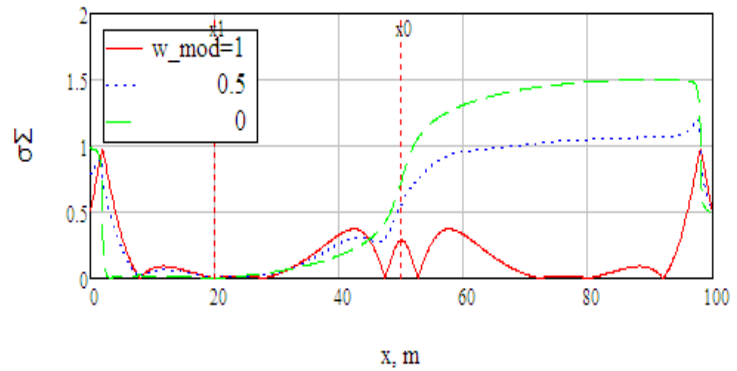

(b)

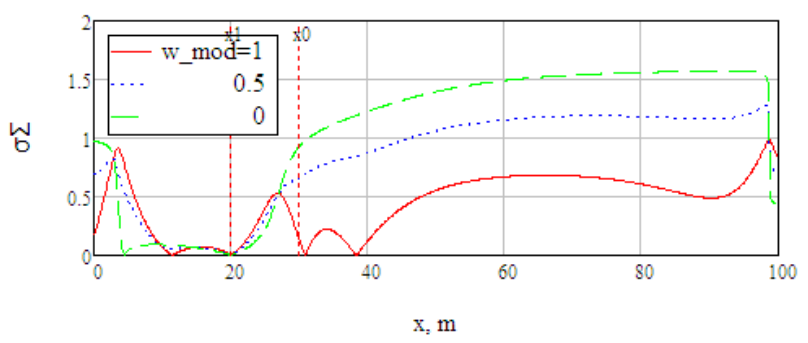

(c)

Figure 4. Localization MSE of the inhomogeneity while displacing along the profile $(x 1=20 \mathrm{~m}$ is the true position of inhomogeneity), (a) Symmetrical installation $\mathrm{x} 0=50 \mathrm{~m}, \varphi \mathrm{AB}=1800$, (b) Symmetrical installation $\mathrm{x} 0=50 \mathrm{~m}, \varphi \mathrm{AB}=900$, (c) Asymmetric installation $\mathrm{x} 0=30 \mathrm{~m}, \varphi \mathrm{AB}=900$

\section{CONCLUSION}

Use of vector measurement systems with registration of electromagnetic field phase characteristics is the basis of the phase-metric method of geoelectric control, which has increased noise immunity. In this paper, application of the compensation method of geoelectric control is done using the phase field characteristics for detection and localization of geodynamic processes on the example of the karst-suffusion cavity. The principle of dynamic positioning control of phase-metric geoelectric control systems, providing increased sensitivity to special changes in the object of study by controlling the sources of probing signals, has been described.

A formalized description of the problem of detection and localization of the near-surface geodynamic process based on the interpretation of the geoelectric section characteristics obtained by observing several electric field parameters using a multipolar installation is developed. Section characteristics are determined by the minimum sum of the weighted mean square interpretation error and the regularizing functional containing a priori information about the geoelectric section.

To verify localization possibility of a spherical karst-suffusion cavity, a simulation of the procedure for interpretation of the section parameters based on the developed technique was carried out. Changes in amplitude and phase of the anomalous field potential component when moving the sphere center along the installation profile for different values of phase shifts between the probing signals is studied. Simulation has shown a good potential differentiation from the heterogeneity position. The smallest value of heterogeneity localization error for both symmetric and asymmetric installation is obtained with simultaneous use of amplitude and phase component in interpretation problem, which indicates the efficiency of combining amplitude and phase methods of geoelectric control of geodynamic processes. 


\section{ACKNOWLEDGEMENTS}

The work is executed under grant of Ministry of education of Russia No. 5.3606.2019/4.6

\section{REFERENCES}

[1] T. Dahlin, "The development of DC resistivity imaging techniques," Computers \& Geosciences, vol. 27, no. 9, pp. 1019-1029, Nov 2001.

[2] Morteza Montazeri-Gh, Mehdi Mahmoodi-k, "Development a new power management strategy for power split hybrid electric vehicles," Transportation Research Part D: Transport and Environment, 37, 79-96, 2015.

[3] J. E. Chambers, et al., "Electrical resistivity tomography applied to geologic, hydrogeologic, and engineering investigations at a former waste-disposal site," Geophysics, vol. 71, no. 6, pp. 231-239. 2006.

[4] A. Kenma, et al., "Complex resistivity tomography for environmental applications," Chemical Engineering Journal, vol. 77, pp. 11-18, 2000.

[5] F. Oliveira and I. Fernandes, "Influence of geotechnical works on neighboring structures," International Multidisciplinary Scientific GeoConference: SGEM: Surveying Geology \& mining Ecology Management, vol. 17, no. 2, pp. 993-1002, 2017.

[6] O. Kuzichkin, et al., "Regression algorithms eliminate interference with the endogenous character geoecological monitoring of water resources," International Multidisciplinary Scientific GeoConference Surveying Geology and Mining Ecology Management, SGEM, vol. 17, pp.749-754, 2017.

[7] O. R. Kuzichkin, "Regression algorithm of formation of predictive geomechanical estimates in geoelectric monitoring," Methods and devices of information transmission and processing, vol. 10. pp. 83-89, 2008.

[8] M. A. Balueva, et al., "Simulation of microcrack influence on temperature variations within geomaterials under deformation," Journal of mining science, vol. 45, pp. 576-580, 2009.

[9] G. S. Vasilyev, et al., "The method of the calibration of the geoelectric systems of the geodynamic control," 18th International Multidisciplinary Scientific GeoConference SGEM, vol. 18, pp. 1011-1018, 2018.

[10] A. Bykov, et al., "Application of a Compensation Inspection Method in Geodynamic Monitoring," In Applied Mechanics and Materials, vol. 799, pp.989-993, 2015.

[11] O. Kuzichkin, et al., "Application of phase-metric measuring system for geodynamic control of karst processes," Journal of Engineering and Applied Sciences, vol. 12, pp. 6858-6563, 2017.

[12] N. V. Dorofeev and O. R. Kuzichkin, "The dynamic management of the parameters of the probing signals at the geodynamic monitoring of the karst processes," 2016 2nd International Conference on Industrial Engineering, Applications and Manufacturing (ICIEAM), Chely abinsk, 2016, pp.1-4.

[13] S. G. Smith, et al., "inventors; AT\&T Delaware Intellectual Property Inc, assignee. Battery voltage indicator in a portable computing device," United States patent US 6, vol. 879, pp. 259. 2005.

[14] W. G. Fateley, et al., "inventors; Plain Sight Systems Inc, assignee. System and method for encoded spatio-spectral information processing," United States patent US 6, vol. 859, pp. 275. 2005.

[15] A. N. Tikhonov and V. Y. Arsenin, "Methods of solving ill-posed problems," Moscow.: Science, 1979.

[16] M. S. Zhdanov, "Electromagnetic geophysics: Notes from the past and the road ahead," Geophysics, vol. 75, no. 5, pp. 7549-7566, 2010.

[17] Oleg R. Kuzichkin, Anastasia Grecheneva, Roman P. Gakhov, Nikolay Dorofeev, Maksim D. Baknin, Bogdan R. Gakhov, "Development and Research of the Geoelectric Model of the Local Zone of Geodynamic Control," Journal of Advanced Research in Dynamical and Control Systems, special issue, issue 13, pp. 620-625, 2018.

[18] E. A. Fainberg, "Controlled Markov processes with arbitrary numerical criteria. Theory of Probability \& Its Applications," vol. 27, no. 3, pp.486-503. 1983.

[19] H. Pape, D. Schweitzer, L. Chen, R. Kutscherauer and M. Walder, "Development of a standard for transient measurement of junction-to-case thermal resistance," Microelectronics Reliability, vol. 52, pp. 1272-1278, 2012.

[20] V. Székely and A. Szalai, "Measurement of the time-constant spectrum: Systematic errors, correction," Microelectron. J, vol. 43, no. 11, pp.904-907, 2012.

[21] M. Wu, et al., "InAlN/GaN heterostructure field-effect transistors on Fe-doped semi-insulating GaN substrates," Journal of Vacuum Science \& Technology B, Nanotechnology and Microelectronics: Materials, Processing, Measurement, and Phenomena, vol. 28, pp.908-911, 2010.

[22] V. Szekely, "Identification of RC networks by deconvolution: chances and limits," in IEEE Transactions on Circuits and Systems I: Fundamental Theory and Applications, vol. 45, no. 3, pp. 244-258, March 1998.

[23] M. Rizwan, et al., "Ideology and Politics of Jamiat Ulema-i-Islam (1947-1973)," Global Social Sciences Review, vol. 3, no. 1, pp. 45-56, 2018.

[24] S. Rauf, et al., "Impact of Electronic Media on Pakistan's Security," Global Social Sciences Review, vol III, no. 1, pp. 434-446, 2018.

[25] S. Panigrahi and A. Thakur, "Modeling and simulation of three phases cascaded H-bridge grid-tied PV inverter," Bulletin of Electrical Engineering and Informatics, vol. 8, no. 1, pp. 1-9, 2019.

[26] M. Nawir, et al., "Effective and efficient network anomaly detection system using machine learning algorithm," Bulletin of Electrical Engineering and Informatics, vol. 8, no. 1, pp. 46-51, 2019. 


\section{BIOGRAPHIES OF AUTHORS}
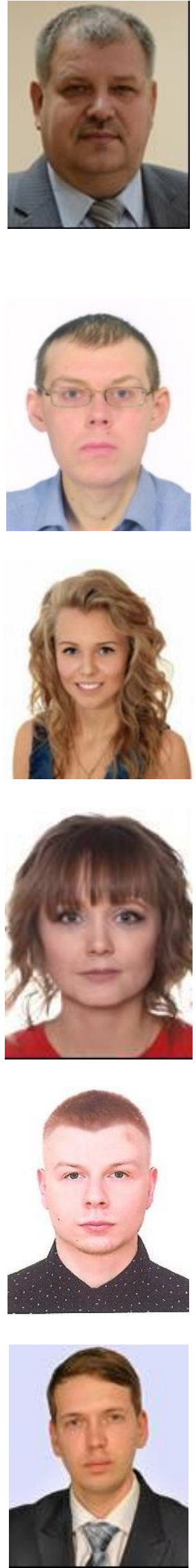

Kuzichkin Oleg Rudolfovich in 1984 received a radio engineer degree from the Vladimir Polytechnic Institute with a degree in Radio Engineering. In 1999 he successfully defended thesis on the topic: "The Monitoring System of Pulsed Geomagnetic Sources" at the Institute of Physics of the Earth, RAS and he was awarded the degree of Candidate of Physical and Mathematical Sciences. In 2009 for the work "Theoretical Foundations of Automated Electromagnetic Control of Geodynamic Objects" he was awarded the degree of Doctor of Technical Sciences. Currently he is a professor at the Department of Information and Robotic Systems at Belgorod State University. Fields of scientific interests: monitoring and diagnostics systems, radio engineering systems, geodynamic monitoring, measuring equipment, electromagnetic signals, information processing, expert systems, automated systems. E-mail: oldolkuz@y andex.ru.

Vasilyev Gleb Sergeevich received a degree in radio engineering from Vladimir state University, Russia, in 2010. He defended the thesis of the candidate of technical Sciences (PhD) in the specialty "Mathematical methods, numerical methods and software packages" in 2017. Since 2017, he has been working as a senior researcher at the Belgorod National Research University, Russia. Research interests include geotechnical and geodynamic monitoring, networks and communication systems, signal generation and conversion devices, frequency synthesizers, and interference compensation. E-mail: vasilievgleb@y andex.ru.

Grecheneva Anastasia Vladimirovna received a degree in "Devices and Methods of Quality Control and Diagnostics" at Vladimir State University, Russia, in 2015. In 2019, she defended her thesis for the degree of candidate of technical sciences (PhD). Currently, she is an assistant professor at Vladimir State University, Russia. Her research interests include automated monitoring systems, geotechnical monitoring, control of geomechanical parameters, measurement methods and tools, and processing heterogeneous data. Email: nastena07_93@mail.ru.

Michaleva Ekaterina Sergeevna received a degree in Quality Management at Oryol State Technical University, Russia, in 2012. She currently works as Deputy Director of the Center for Scientific Communication and Exhibition Activities of the Department of Scientific Communication and Publishing at the Belgorod National Research University, Russia. Her research interests include automated monitoring systems, geotechnical monitoring, control of geomechanical parameters, measurement methods and tools, and processing heterogeneous data. Email: pankina@bsu.edu.ru.

Baknin Maxim Dmitrievich in 2015 received a bachelor's degree in Instrument Engineering at Vladimir State University, Russia, in 2017 - a master's degree in this specialty. He is currently a graduate student at Belgorod National Research University, Russia, in the field of training "Informatics and Computer Engineering" under the program "Automation and Control of Technological Processes and Production (by Industry)". His research interests include engineering geology, oil and gas geology, and seismoacoustic effects in geology. Email: m.baknin@yandex.ru.

Surzhik Dmitry Igorevich received a degree in radio engineering from Vladimir State University, Russia, in 2012. He defended the thesis of the candidate of technical sciences (PhD) in the specialty "Radio engineering, including television systems and devices" in 2017. From 2017 to the present, he has been working as an assistant professor at Vladimir State University, Russia. His research interests include geodynamic monitoring, communication networks and systems, signal generation and conversion devices, frequency synthesizers, interference compensation, and approximation methods. Email: arzerum@mail.ru. 